Density profile peaking in JET H-mode plasmas: experiments versus linear gyrokinetic predictions

This article has been downloaded from IOPscience. Please scroll down to see the full text article.

2009 Nucl. Fusion 49075037

(http://iopscience.iop.org/0029-5515/49/7/075037)

The Table of Contents and more related content is available

Download details:

IP Address: 128.178.183.125

The article was downloaded on 08/04/2010 at 10:04

Please note that terms and conditions apply. 


\title{
Density profile peaking in JET H-mode plasmas: experiments versus linear gyrokinetic predictions
}

\author{
M. Maslov ${ }^{1}$, C. Angioni ${ }^{2}$, H. Weisen ${ }^{1}$ and JET-EFDA \\ contributors $^{\mathrm{a}}$ \\ JET-EFDA, Culham Science Centre, Abingdon, OX14 3DB, UK \\ ${ }^{1}$ Ecole Polytechnique Fédérale de Lausanne (EPFL), Centre de Recherches en Physique des \\ Plasmas, Association Euratom-Confédération Suisse, CH-1015 Lausanne, Switzerland \\ ${ }^{2}$ Max-Planck-Institut für Plasmaphysic, Association Euratom, D-85748 Garching, Germany \\ E-mail: mikhail.maslov@epfl.ch, clemente.angioni@ipp.mpg.de and henri.weisen@epfl.ch
}

Received 24 December 2008, accepted for publication 19 May 2009

Published 6 July 2009

Online at stacks.iop.org/NF/49/075037

\begin{abstract}
As an independent complement to previous studies (Weisen et al 2005 Nucl. Fusion 45 L1-4, Weisen et al 2006 Plasma Phys. Control. Fusion 48 A457-66, Angioni et al 2007 Nucl. Fusion 47 1326-35), density peaking in the JET tokamak was investigated on the dataset, comprising virtually all H-mode experiments performed in 2006-2007. Unlike previous studies, this work focuses on low collisionality data as most representative of reactor conditions. The study confirms that collisionality is the most important parameter governing density peaking in H-mode, followed by the NBI particle flux and/or the $T_{\mathrm{i}} / T_{\mathrm{e}}$ temperature ratio. For the first time in JET a modest, albeit significant dependence of peaking on internal inductance, or magnetic shear is seen. The experimental behaviour is compared with an extensive database of linear gyrokinetic calculations using the GS2 code. The predictions from GS2 simulations based on the highest linear growth rate mode are in good agreement with experimental observations. They are also corroborated by initial results from the non-linear code GYRO.
\end{abstract}

PACS numbers: 52.55.Fa, 52.65.Tt, 52.25.Fi

(Some figures in this article are in colour only in the electronic version)

\section{Introduction}

The degree of peaking of the density profile has profound implications for reactor operation. A burning plasma with a peaked density profile has a potential for higher fusion power output and higher bootstrap current. At the same time, a peaked density profile may lead to impurity accumulation with a negative impact on performance. Peaked density profiles were observed in many tokamaks [1-6] and so far there is no comprehensive model able to describe all aspects of particle transport.

This paper pursues work previously undertaken on AUG and JET [2-4], which has shown that collisionality is the most important scaling parameter for density peaking in $\mathrm{H}$-modes. It uses an entirely new experimental dataset, obtained from experiments performed in 2006 and 2007. One of the key diagnostics, LIDAR Thomson scattering, was recalibrated

a See the appendix of Romanelli F. et al 2008 Proc. 22nd Int. Fusion Energy Conf. 2008 (Geneva, Switzerland, 2008) (Vienna: IAEA). since the earlier work and we have used different inversion algorithms for inverting far infrared interferometer data to avoid any possible bias introduced by the inversion methods. In previous work, the neutral beam source emerged as a second most important parameter influencing density peaking. The influence of several other parameters was investigated too, without providing a definite conclusion. The aim of this study is to revisit all parameter dependences on an independent database, with emphasis on low collisonalities and to provide a comparison with predictions from quasi-linear and non-linear gyrokinetic theory.

For consistency with previous publications, we keep most of the important definitions the same. The effective collisionality is defined as $v_{\mathrm{eff}}=v_{\mathrm{ei}} / \omega_{\mathrm{d}}=v_{\mathrm{ei}} / 2 k_{\mathrm{y}} \rho_{\mathrm{s}}\left(c_{\mathrm{s}} / R\right)=$ $10^{-14} Z_{\text {eff }} R n_{\mathrm{e}} /\left\langle T_{\mathrm{e}}\right\rangle^{2}$, where $v_{\mathrm{e}}$ is electron collisionality, $\omega_{\mathrm{d}}$ is the curvature drift frequency, $\left\langle n_{\mathrm{e}}\right\rangle$ and $\left\langle T_{\mathrm{e}}\right\rangle$ are volume averaged electron density and temperature. The normalized mode number of the most unstable mode is estimated as $k_{\theta} \rho_{\mathrm{s}} \approx \sqrt{0.1}$, where $c_{\mathrm{s}}=\sqrt{T_{\mathrm{e}} / m_{D}}$ is the ion sound speed. 

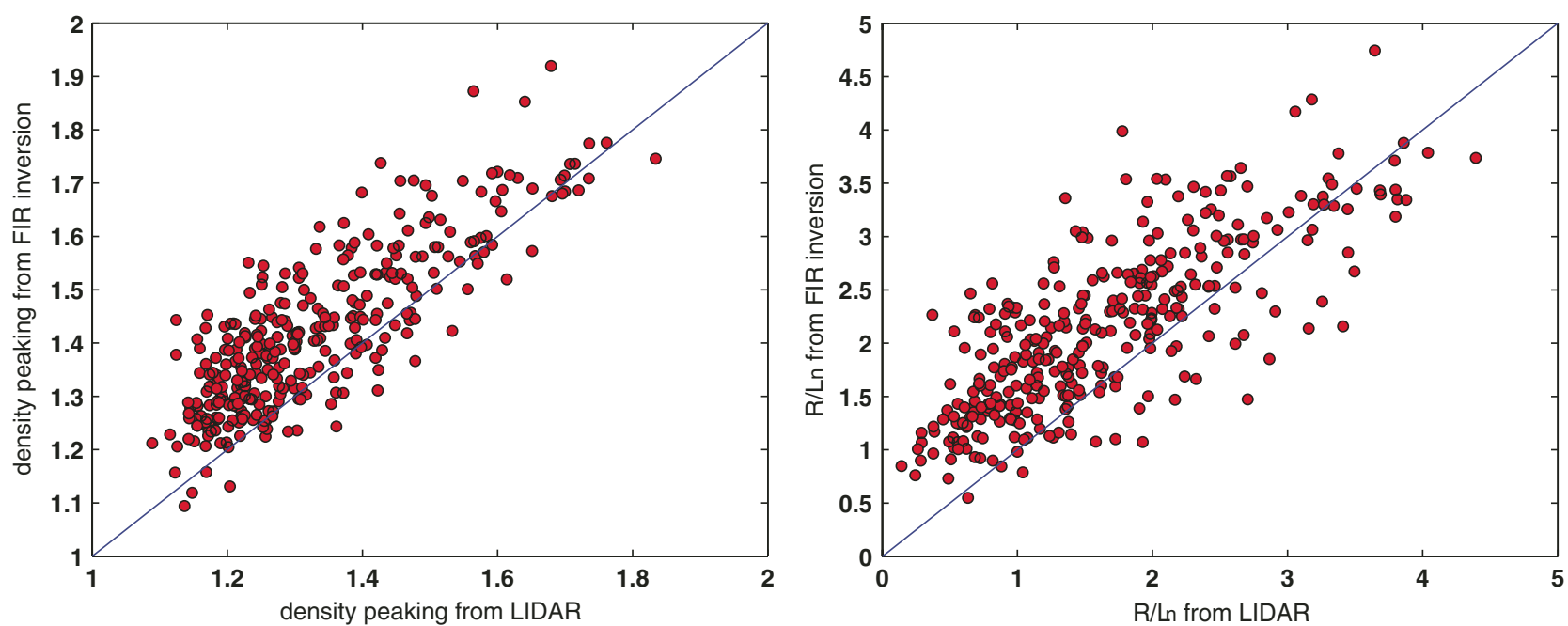

Figure 1. Comparison between LIDAR and interferometer density profile measurements $\left(n_{0.2} /\left\langle n_{\mathrm{e}}\right\rangle\right.$ for the left one, $R / L_{\mathrm{n}}$ at $r / a=0.5$ for the right one).

We assume $Z_{\text {eff }}=2$ for all cases, due to the uncertainties with the measurements of $Z_{\text {eff }}$ in the experiments, leaving $v_{\text {eff }}=2 \times 10^{-14} R\left\langle n_{\mathrm{e}}\right\rangle /\left\langle T_{\mathrm{e}}\right\rangle^{2}$ as the working definition for $v_{\text {eff }}$ here.

As a measure of density peaking, we use the ratio of average density inside the $r / a=0.2$ surface and overall volume average density, $n_{0.2} /\left\langle n_{\mathrm{e}}\right\rangle$. We also use the normalized density gradient $R / L_{\mathrm{n}}$ at $r / a=0.5$. The minor radius is defined as the flux surface half width at the equatorial plane and the gradient was calculated by a linear fit over the range $r / a=0.2-0.8$. Taking such a wide region can result in an underestimation of normalized density gradient with respect to the real local value at mid-radius, since that range often overlaps with the flat density profile region usually observed inside the $q=1$ surface. However that choice is justified by a minimization of error bars, caused by limitations of the diagnostic capabilities in measuring local density values. $R / L_{\mathrm{n}}$ must be used for comparison with theoretical predictions from the local flux tube code GS2. We also present scalings using the density peaking parameter $n_{\mathrm{e} 0} /\left\langle n_{\mathrm{e}}\right\rangle$ since this is a common parameter for intermachine comparisons. Both these values were derived from measured profiles and stored in our database.

The dimensionless particle source is defined as $\Gamma^{\prime}=$ $e T_{\mathrm{i}} S / Q_{\mathrm{i}}$, where $S\left(\mathrm{~m}^{-2} \mathrm{~s}^{-1}\right)$ is the particle flux and $Q_{\mathrm{i}}\left(\mathrm{W} \mathrm{m}^{-2}\right)$ is the ion heat flux through a given flux surface (here always taken at $r / a=0.5), e$ is the electron charge and $T_{\mathrm{i}}$ is the local ion temperature. This definition differs from the one used in previous publications $[3,4], \Gamma^{*}=R S_{\mathrm{NBI}} / n_{\mathrm{e}} \chi$, where $\chi$ is the heat diffusivity. The definition adopted here makes the comparison with simulations straightforward, as explained later. These two parameters, $\Gamma^{\prime}$ and $\Gamma^{*}$, are highly correlated in our database, so the general conclusions of the analysis remain independent of the definition used.

\section{Experiments}

For the analysis of experimental data we have chosen $\sim 270$ plasma discharges performed on the JET tokamak in 2006 and 2007. All samples are from H-modes with stationary density, as measured using the FIR interferometer, for at least $1 \mathrm{~s}$ duration. On the basis of these experiments a comprehensive profile database was created, which allows us to study dependences between various plasma parameters and their influence on density peaking. Two independent measurements of density profile were used: LIDAR Thomson scattering and inversion of the line integrated density from the far infrared interferometer (six chords covering the whole plasma section). Those two diagnostics provide consistent results for both the peaking factor and the density gradient measurements as shown in figure 1. The interferometer provides on average slightly higher values, but the discrepancy is small and independent of operating conditions. For further analysis we will use only the less scattered interferometer inversion data. Using the LIDAR measurements does not significantly change the parameter dependences of the fits, as already shown in [3], nor the conclusions drawn from this work.

All of the discharges in this dataset are dominantly NBI heated. About $20 \%$ of the discharges also had some LHCD heating and $50 \%$ of them had additional ICRH heating, which allowed us to obtain a variation of the $l_{\mathrm{i}}$ and $T_{\mathrm{i}} / T_{\mathrm{e}}$ parameters.

NBI power and particle deposition in the JET plasma core are calculated using the PENCIL code [7]. Calculations are routinely available for every shot, but PENCIL does not take into account penetrating edge neutrals, nor halo neutrals, which are born in the plasma core as a result of charge exchange of beam neutrals and thermal ions. More accurate calculations using TRANSP [8], including edge and halo neutrals, are available only for a limited number of discharges. Comparing the particle sources estimated by TRANSP and PENCIL inside $r / a=0.5$, we found that the difference does not exceed $20 \%$ and for all the existing cases with both code results available, NBI fuelling is significantly higher than penetration of neutrals from the edge in the central plasma region. For the database we therefore only use the NBI fuelling calculated routinely by the PENCIL code.

It has already been shown in earlier experiments that density peaking in $\mathrm{H}$-mode plasma scales with the logarithm of the effective collisionality [1-6]. The same tendency is also 

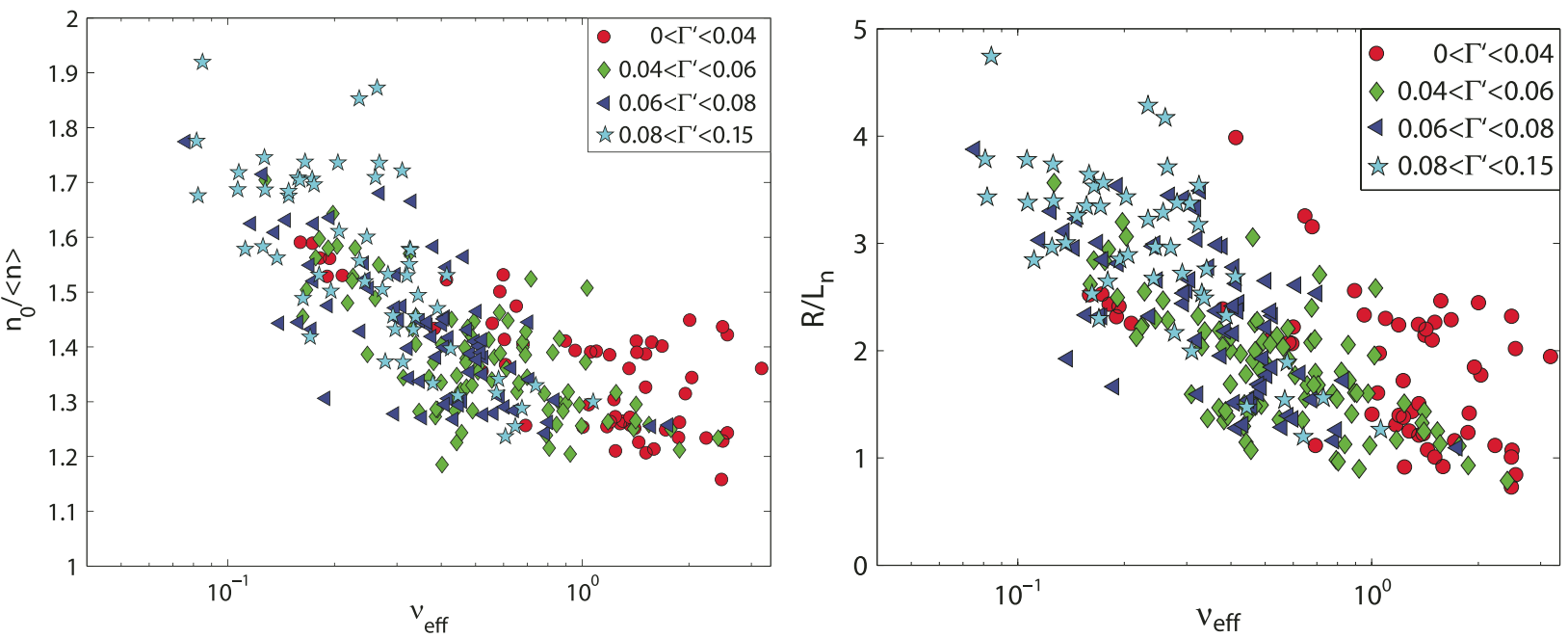

Figure 2. Density peaking (left) and normalized density gradient (right) as a function of the effective collisionality from interferometer measurements.

clearly seen in the recent JET plasmas studied in this paper (see figure 2) with an additional feature: around $\nu_{\text {eff }} \sim 0.5$ there appears to be an inflexion point where the collisionality dependence disappears, which is well visible on the $n_{0.2} /\left\langle n_{\mathrm{e}}\right\rangle$ measurement and a little less pronounced on $R / L_{\mathrm{n}}$. The reason for this behaviour may lie in the limited diagnostics resolution for measuring the edge part (pedestal) of the density profile. We limit our analysis to the low collisionality branch $\left(v_{\text {eff }}<0.5\right)$, which represents the monotonic $v_{\text {eff }}^{1 / 2}$ dependence and provides the relevant domain for ITER extrapolations.

The purpose of this experimental database analysis is to find the empirical relation(s) between density peaking and other dimensionless plasma parameters. We focus on the following 10 variables: $v_{\text {eff }}, \Gamma^{\prime}, \rho^{*}$ (the normalized Larmor radius), $T_{\mathrm{i}} / T_{\mathrm{e}}, q_{95}$ (safety factor at $95 \%$ of poloidal flux), $\beta_{\mathrm{N}}$, $R / L_{T_{\mathrm{e}}}, R / L_{T_{\mathrm{i}}}, l_{\mathrm{i}}$ (internal inductance obtained from magnetic measurements) and $\delta$ (triangularity at the last closed flux surface). The temperature gradients were obtained with linear fit over $r / a=0.3-0.7$, by analogy with $R / L_{\mathrm{n}}$, but over a slightly shorter stretch. For the local values, an average over the same interval was used. The histograms in figure 3 show their variations in these experiments. They are well spread, which allows us to study their respective bivariate correlations (see table 1) for the low collisionality part of the database $\left(v_{\text {eff }}<0.5\right)$. In this work we will use the square root of effective collisionality instead of the logarithm. For the empirical analysis that substitution does not produce a significant difference, but it will be easier for the comparison with simulation results presented later in the paper.

As one can see, density peaking is strongly correlated with $v_{\text {eff }}^{1 / 2}$. The parameters $\Gamma^{\prime}, T_{\mathrm{i}} / T_{\mathrm{e}}$ and $\delta$ show a moderate correlation, $\beta_{\mathrm{N}}$ and $l_{\mathrm{i}}$ have the lowest (barely visible) correlation with density gradients and the other parameters $\left(q_{95}, \rho^{*}, R / L_{T_{\mathrm{e}}}, R / L_{T_{\mathrm{i}}}\right)$ are not relevant at all, confirming conclusions from an earlier dataset on JET [3]. For constructing the scaling expressions of $n_{0.2} /\left\langle n_{\mathrm{e}}\right\rangle$ and $R / L_{\mathrm{n}}$ we will use only those parameters which are highly or moderately correlated with density peaking. We also keep $l_{i}$ in spite of the low cross-correlation, since this parameter is reported to be significant for density peaking in L-mode plasmas [9]. The mean values and standard deviation of each of the regression variables are shown in table 2 .

For multivariate regression analysis we will use linear fits of the form $Y=c+\operatorname{sum}\left(\left(a_{\mathrm{j}} \pm \sigma\left(a_{\mathrm{j}}\right)\right) \cdot X_{\mathrm{j}}\right)$, where $Y$ is the regressed variable, $X_{\mathrm{j}}$ are the regression variables (those listed in the table above), $a_{\mathrm{j}}$ are the regression coefficients and $\sigma\left(a_{\mathrm{j}}\right)$ are the standard deviations of $a_{\mathrm{j}}$ and correspond to a $66 \%$ confidence interval. The statistical significance is defined as $\mathrm{StS}=a_{\mathrm{j}} / \sigma\left(a_{\mathrm{j}}\right)$ and measures the importance of a parameter in particular scaling. StS $<1$ means that the corresponding parameter is irrelevant for the considered regression and excluding it from the fit will not change its quality.

The RMS (root mean square deviation) quantifies the difference between the real database values and values predicted by the regression formula. The lowest obtainable value corresponds to measurements error of the regressed variables. For the density peaking factor it can be roughly estimated as $\sim 0.1$.

The linear regression of density peaking with all possible parameter combinations are shown in the appendix. The single parameter fit with the lowest RMS error and the most significant regression variable is $n_{0.2} /\left\langle n_{\mathrm{e}}\right\rangle=(1.96 \pm 0.04)-$ $(0.88 \pm 0.07) v_{\text {eff }}^{1 / 2}$. Two parameter fits with combinations of $\left\{v_{\text {eff }}^{1 / 2} ; \Gamma^{\prime}\right\}$ and $\left\{v_{\text {eff }}^{1 / 2} ; T_{\mathrm{i}} / T_{\mathrm{e}}\right\}$ produce regressions of similar quality. This is easily understood from the high degree of correlation observed between $\Gamma^{\prime}$ and $T_{\mathrm{i}} / T_{\mathrm{e}}$ (a coefficient of 0.93 in table 1 , the highest number obtained), which is common for all NBI heated plasmas. The reason is in the properties of electron and ion heating produced by thermalizing beam particles. In our definition, $\Gamma^{\prime}=S / Q_{\mathrm{i}} T_{\mathrm{i}}, S$ represents the total particles flux and $Q_{\mathrm{i}}$ is the total ion heat flux from the plasma core. For all cases considered in our database, the majority of particles delivered inside $r / a=0.5$ surface are produced by neutral beams (thus neglecting edge neutral penetration) and ion heating is provided mainly by NBI. Although the equipartitioning heat flux between electrons and ions is taken into account for calculating $Q_{\mathrm{i}}$, it is less than $20 \%$ of the NBI ion heating for all the cases in our database except a very few which do not affect the overall statistics. So $Q_{\mathrm{i}} / S \sim Q_{\mathrm{i}} / Q_{\text {tot }} \sim E_{\text {crit }} / E_{\text {beam }}$ [10], where $E_{\text {beam }}$ is the 

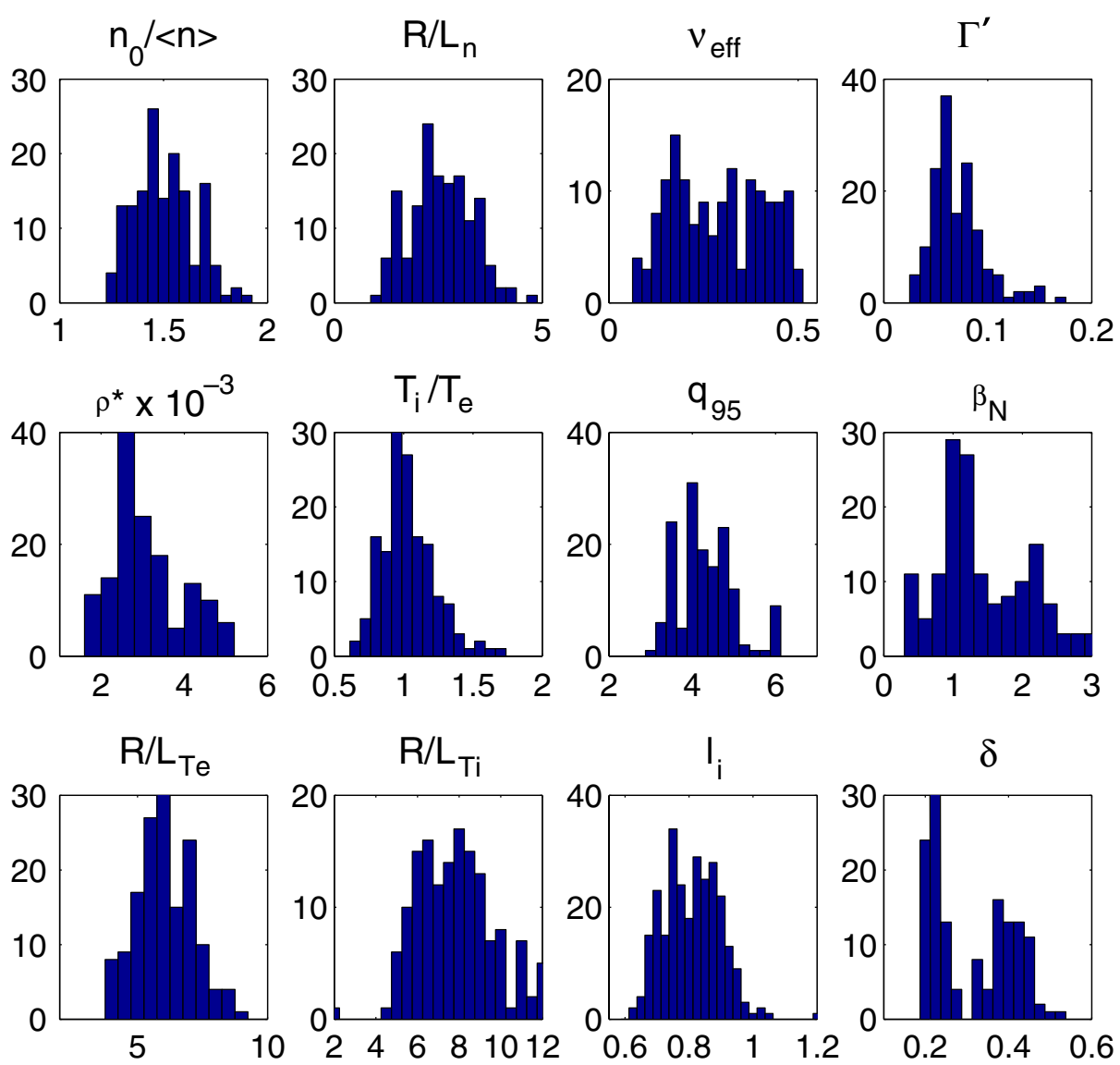

Figure 3. Number of observations per value for each of the dimensionless parameters in the experimental dataset $\left(v_{\text {eff }}<0.5\right)$.

Table 1. Cross-correlation coefficients between various experimental parameters $\left(v_{\text {eff }}<0.5\right)$.

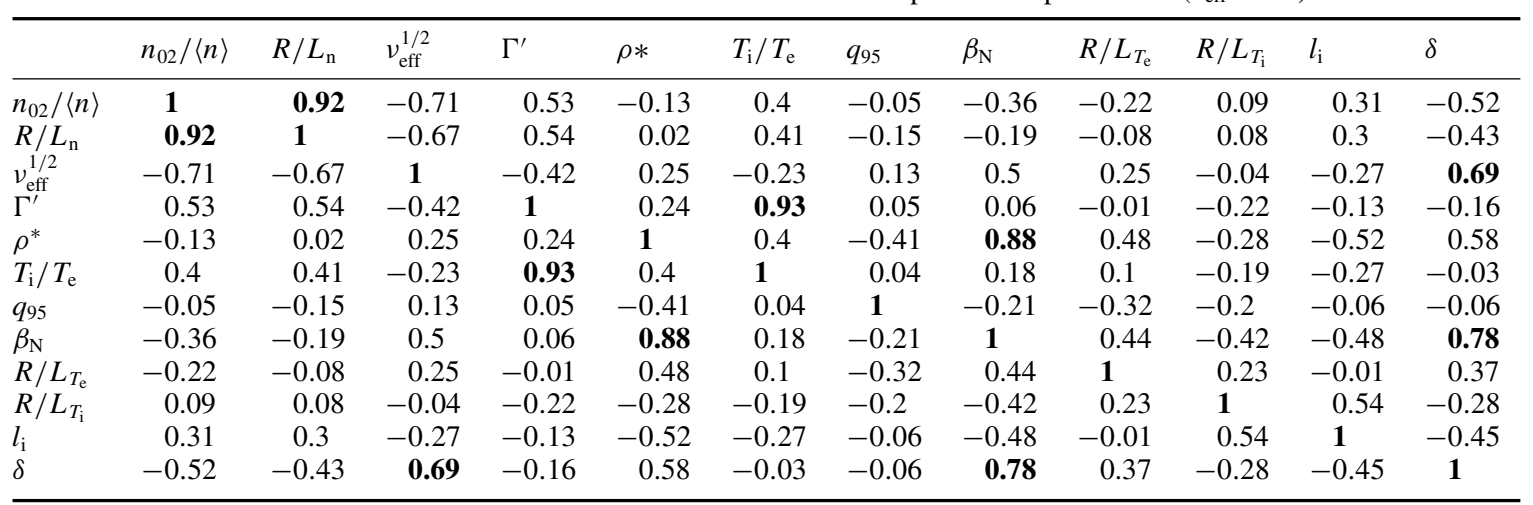

energy of the neutral beam particles and $E_{\text {crit }}$ is the critical energy.

$$
E_{\text {crit }}=14.8 T_{\mathrm{e}}\left[\frac{A^{3 / 2}}{n_{\mathrm{e}}} \sum \frac{n_{j} Z_{j}^{2}}{A_{j}}\right]^{2 / 3} \sim \text { const } \cdot T_{\mathrm{e}},
$$

while the launched neutral particles energy remains independent of plasma conditions, so $Q_{\mathrm{i}} / S \sim T_{\mathrm{e}} / E_{\text {beam }}$ and $\Gamma^{\prime}=S / Q_{\mathrm{i}} T_{\mathrm{i}}=T_{\mathrm{i}} / T_{\mathrm{e}} \cdot E_{\text {beam }} \sim$ const $\cdot T_{\mathrm{i}} / T_{\mathrm{e}}$.

As a consequence, regressions which include $T_{\mathrm{i}} / T_{\mathrm{e}}$ and $\Gamma^{\prime}$ together in this dataset are not relevant, since one of these parameters (or both) loses its statistical significance. Including only one of the two generally increases the quality of the fit. We conclude that at least one of these parameters is important for
Table 2. Mean values and standard deviations of scaling parameters.

\begin{tabular}{llllll}
\hline & $\nu_{\text {eff }}^{1 / 2}$ & $\Gamma^{\prime}$ & $T_{\mathrm{i}} / T_{\mathrm{e}}$ & $\delta$ & $l_{\mathrm{i}}$ \\
\hline\langle\rangle, mean value & 0.53 & 0.071 & 1.03 & 0.30 & 0.79 \\
$\sigma$, standard deviation & 0.13 & 0.026 & 0.19 & 0.10 & 0.088 \\
\hline
\end{tabular}

the density peaking, but it is impossible to decorrelate them in this particular database since one would need discharges with dominant electron heating (or ion heating of other than NBI nature). For the experiments considered in this paper there are no such data unfortunately.

Inclusion of $\delta$ together with collisionality zeroes the statistical significance of the former in 2, 3 and 4 parameter 
fits, so we can conclude that the effect of $\delta$ on density peaking is caused primarily by its correlation with $v_{\text {eff }}$ (factor 0.69 in table 1). This correlation results from the plasma operational scenario specifications, rather than from physics (H-modes with higher triangularity normally have higher densities and higher collisionalities).

Among three-parameter fits, the best ones combine $\left\{v_{\text {eff }}^{1 / 2} ; \Gamma^{\prime} ; l_{\mathrm{i}}\right\}$ and $\left\{v_{\text {eff }}^{1 / 2} ; T_{\mathrm{i}} / T_{\mathrm{e}} ; l_{\mathrm{i}}\right\}$. The internal inductance is statistically significant in all the cases with a regression coefficient in the range $\sim 0.3-0.4$. We can conclude that in our dataset $l_{i}$ does have a modest effect on density peaking.

In all the fits $v_{\text {eff }}^{0.5}$ has the highest statistical relevance: $\mathrm{StR}=a\left(v_{\mathrm{eff}}^{1 / 2}\right) \cdot \sigma\left(v_{\mathrm{eff}}^{1 / 2}\right) \sim 0.07$, which is at least two times higher than those for $T_{\mathrm{i}} / T_{\mathrm{e}}, \Gamma^{\prime}$ and $l_{\mathrm{i}}$.

As a conclusion from the experimental database analysis, the effective collisionality is the principal parameter related to density gradient formation, followed by $T_{\mathrm{i}} / T_{\mathrm{e}}$ and/or $\Gamma^{\prime}$. The effect of internal inductance is significant, but of much lesser magnitude than that of $v_{\text {eff }}$.

The best empirical scalings obtained here for the peaking factor are

$$
\begin{aligned}
& n_{0.2} /\left\langle n_{\mathrm{e}}\right\rangle=1.96 \pm 0.04-(0.88 \pm 0.07) v_{\mathrm{eff}}^{1 / 2}, \\
& n_{0.2} /\left\langle n_{\mathrm{e}}\right\rangle=1.78 \pm 0.05-(0.74 \pm 0.07) v_{\mathrm{eff}}^{1 / 2} \\
& \quad+(1.58 \pm 0.32) \Gamma^{\prime} \\
& n_{0.2} /\left\langle n_{\mathrm{e}}\right\rangle=1.74 \pm 0.05-(0.81 \pm 0.07) v_{\mathrm{eff}}^{1 / 2} \\
& \quad+(0.18 \pm 0.04) T_{\mathrm{i}} / T_{\mathrm{e}} \\
& \quad+(1.92 \pm 0.32) \Gamma^{\prime}+(0.34 \pm 0.09) l_{\mathrm{i}}, \\
& n_{0.2} /\left\langle n_{\mathrm{e}}\right\rangle=1.43 \pm 0.10-(0.63 \pm 0.07) v_{\mathrm{eff}}^{1 / 2} \\
& \quad+(0.25 \pm 0.04) T_{\mathrm{i}} / T_{\mathrm{e}}+(0.39 \pm 0.09) l_{\mathrm{i}} .
\end{aligned}
$$

The best empirical scalings obtained for the normalized gradient are

$$
\begin{aligned}
& R / L_{\mathrm{n}}=4.59 \pm 0.21-(3.95 \pm 0.38) v_{\mathrm{eff}}^{1 / 2} \\
& R / L_{\mathrm{n}}=3.33 \pm 0.27-(2.99 \pm 0.38) v_{\mathrm{eff}}^{1 / 2}+(10.6 \pm 1.7) \Gamma^{\prime} \\
& R / L_{\mathrm{n}}=3.07 \pm 0.33-(3.47 \pm 0.36) \nu_{\mathrm{eff}}^{1 / 2} \\
& \quad+(1.23 \pm 0.21) T_{\mathrm{i}} / T_{\mathrm{e}}
\end{aligned}
$$

$$
\begin{aligned}
& R / L_{\mathrm{n}}=2.32 \pm 0.56-(2.69 \pm 0.40) v_{\mathrm{eff}}^{1 / 2}+(11.59 \pm 1.75) \Gamma^{\prime} \\
& \quad+(0.99 \pm 0.49) l_{\mathrm{i}},
\end{aligned}
$$$$
R / L_{\mathrm{n}}=1.67 \pm 0.64-(3.12 \pm 0.38) v_{\mathrm{eff}}^{1 / 2}
$$$$
+(1.44 \pm 0.23) T_{\mathrm{i}} / T_{\mathrm{e}}+(1.28 \pm 0.51) l_{\mathrm{i}} .
$$

For a comparison with simulations we will need to know the relation between $\Gamma^{\prime}$ and $T_{\mathrm{i}} / T_{\mathrm{e}}$, which can be found directly from the equations above or via a separate regression calculation:

$$
\begin{aligned}
& \Gamma^{\prime}=-(0.027 \pm 0.008)-(0.045 \pm 0.009) v_{\mathrm{eff}}^{1 / 2} \\
& \quad+(0.118 \pm 0.006) T_{\mathrm{i}} / T_{\mathrm{e}} \text { or } \\
& T_{\mathrm{i}} / T_{\mathrm{e}}=(0.34 \pm 0.06)+(0.30 \pm 0.08) v_{\mathrm{eff}}^{1 / 2} \\
& \quad+(7.58 \pm 0.36) \Gamma^{\prime}
\end{aligned}
$$

To make an empirically based prediction for ITER density profile, we assume $\left\langle n_{\mathrm{e}}\right\rangle=10^{20} \mathrm{~m}^{-3},\left\langle T_{\mathrm{e}}\right\rangle=8 \mathrm{keV}, \Gamma^{\prime}=0$, $T_{\mathrm{i}} / T_{\mathrm{e}}=1$, which corresponds to the inductive reference scenario [11]. That gives us $v_{\text {eff }}$ (ITER) $=0.19$ and $n_{0.2} /\left\langle n_{\mathrm{e}}\right\rangle(\mathrm{ITER})=1.42-1.55, R / L_{\mathrm{n}}(\mathrm{ITER})=2.0-2.8$, depending which of the derived scaling expressions is used. The prediction for the peaking factor is in agreement with the extrapolations done in previous publications [2-4] despite being based on different datasets and using slightly different regression variables than here. The expectations for $R / L_{\mathrm{n}}$ values are lower in this work $(2.4 \pm 0.4$ instead of $4.0 \pm 1.0)$, but as stated before, the value used in this work is calculated over the large density profile range and can be slightly biased with respect to definitions used in other publications.

\section{GS2 simulations}

We have evaluated the role of microturbulence predicted by linear gyrokinetic theory using a large number $(>1000)$ of linear gyrokinetic simulations produced with the GS2 flux tube code $[12,13]$ with input parameters representative of the experimental parameters. All simulations were done for $r / a=0.5$ with fixed $R / L_{T_{\mathrm{e}}}=6.0, q=1.5, \hat{s}=0.45$, $\kappa=1.45, \kappa^{\prime}=0.1, \delta=0.12, \delta^{\prime}=0.06$. The plasma shape parameters match the average values for $r / a=0.5$ in the JET experimental database, discussed in the previous section. While keeping these values constant, we independently varied four other parameters: $R / L_{\mathrm{n}}, R / L_{T_{\mathrm{i}}}, T_{\mathrm{i}} / T_{\mathrm{e}}$ and $\nu_{\mathrm{eff}}$. Since the simulations are linear, perturbed quantities grow indefinitely without saturation. However the ratio of particle flux and ion heat flux generated (which we denote as $\Gamma_{\mathrm{GS} 2}$ ) remains constant since it depends only on the mode structure and not on its final amplitude. This dimensionless value (ion heat flux is normalized by ion temperature) is the result of each particular GS2 run.

Simulations were done for 10 values of $k_{\mathrm{y}}=0.1$, $0.2, \ldots, 1.0$ and the heat and particle fluxes generated were calculated for the mode with the highest linear growth rate (the fastest mode number varies between 0.3 and 0.6 for different plasma conditions). Grid parameters in the GS2 input (ngauss $=10$, negrid $=16$, ecut $=6.0$, ntheta $=64$, nperiod $=8$ ) are ensuring a good convergency for each of the wavenumber chosen.

The real mode frequency in our simulations was always in the ion drift direction, which allows us to identify them as ITG modes.

The definitions for collisionality used in GS2 differ from the convention used in the experimental database. The GS2 definition $v_{\mathrm{GS} 2}=v_{\mathrm{e}}\left(\sqrt{2} c_{\mathrm{s}} / R\right)^{-1}$ differs by a factor of $\sqrt{0.2}$ from the definition of $v_{\text {eff }}$. Another difference arises from the fact that in the empirical database we used volume averaged 

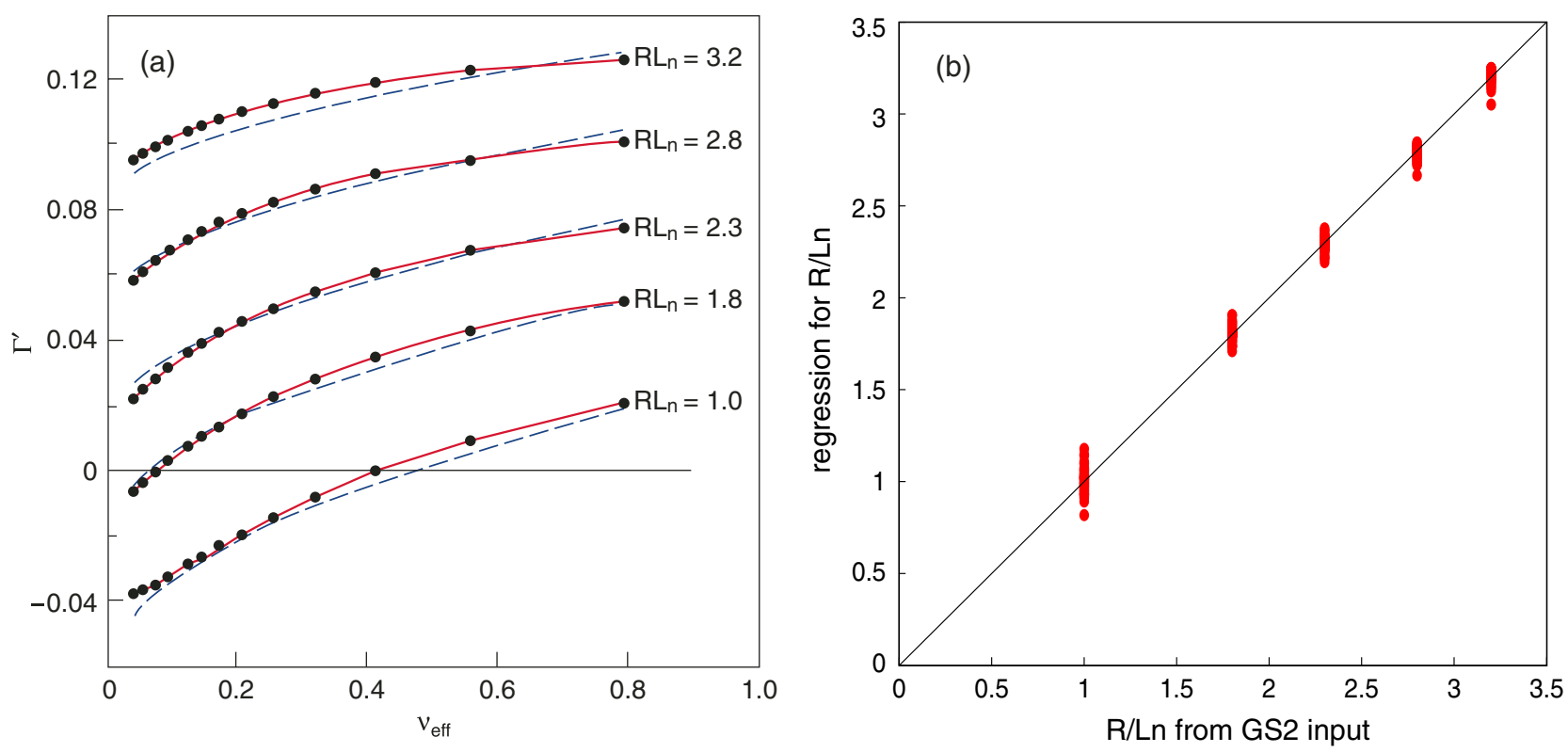

Figure 4. (a) GS2 simulation results for $T_{\mathrm{i}} / T_{\mathrm{e}}=1, R / L_{T_{\mathrm{i}}}=R / L_{T_{\mathrm{e}}}=6.0$ Dashed line-approximation of $\Gamma_{\mathrm{GS} 2}$ with expression (4), (b) regressed values (expression (5)) of $R / L_{\mathrm{n}}$ versus those used in GS2 input.

$\left\langle n_{\mathrm{e}}\right\rangle$ and $\left\langle T_{\mathrm{e}}\right\rangle$, while in the simulations we assumed local values at $r / a=0.5$. The local and volume average effective collisionalities in the database are very well correlated and differ by a factor near 0.43: $v_{r / a=0.5} \approx 0.43 v_{\text {eff }}$, which gives the final expression for conversion from GS2 to JET collisionality: $v_{\mathrm{GS} 2}=0.192 v_{\mathrm{eff}}$. To avoid confusion we only use the experimental definition of effective collisionality, as defined in the introduction.

For the first group of simulations we used 13 values of $v_{\text {eff }}$ between 0.04 and $0.8,4$ values of $T_{\mathrm{i}} / T_{\mathrm{e}}(0.75,0.85,1.0$, $1.15), 5$ values of $R / L_{\mathrm{n}}(1.0,1.8,2.3,2.8,3.2)$ and 4 values of $R / L_{T_{\mathrm{i}}}(6.0,7.0,8.0,9.0)$. All these parameters were varied independently, requiring $1040 \mathrm{GS} 2$ runs in total. To facilitate the data analysis, we will split these simulations into four groups, corresponding to different $R / L_{T_{\mathrm{i}}}$ values and consider them separately.

In each of those groups, $\Gamma_{\mathrm{GS} 2}$ (which is the result of every single run) is presented as a table function of the other three parameters: $v_{\mathrm{eff}}, T_{\mathrm{i}} / T_{\mathrm{e}}$ and $R / L_{\mathrm{n}}$ (see figure $4(a)$ ). To facilitate our comparison with the experimental results, we introduce an approximation for $\Gamma_{\mathrm{GS} 2}$. It was found that $\Gamma_{\mathrm{GS} 2}$ scales linearly with $T_{\mathrm{i}} / T_{\mathrm{e}},\left(v_{\mathrm{eff}}\right)^{1 / 2}$ and $\left(R / L_{\mathrm{n}}\right)^{3 / 2}$. The collisionality dependence itself is dependent on $R / L_{\mathrm{n}}$, as can be seen on figure 4(a) (the solid lines connecting the fit results are steeper for lower gradient values). We find that introducing the nonlinear term $\left(v_{\mathrm{eff}}\right)^{1 / 2}\left(R / L_{\mathrm{n}}\right)^{3 / 2}$ along with the previous allows us to obtain a satisfactory multiparameter regression for $\Gamma_{\mathrm{GS} 2}$ :

$$
\begin{aligned}
\Gamma_{\mathrm{GS} 2} & =-0.146+\left(0.0305-0.0081 v_{\text {eff }}^{1 / 2}\right)\left(R / L_{\mathrm{n}}\right)^{3 / 2} \\
& +0.1 v_{\text {eff }}^{1 / 2}+0.0524 T_{\mathrm{i}} / T_{\mathrm{e}} .
\end{aligned}
$$

The dashed lines in figure $4(a)$ correspond to $\Gamma_{\mathrm{GS} 2}$ obtained from this simple fit. From this expression we can extract $R / L_{\mathrm{n}}$ as a function of other parameters:

$$
R / L_{\mathrm{n}}=\left(\frac{0.146+\Gamma_{\mathrm{GS} 2}-0.0524 T_{\mathrm{i}} / T_{\mathrm{e}}-0.1 \sqrt{\nu_{\mathrm{eff}}}}{0.0305-0.0081 \sqrt{\nu_{\mathrm{eff}}}}\right)^{2 / 3} .
$$

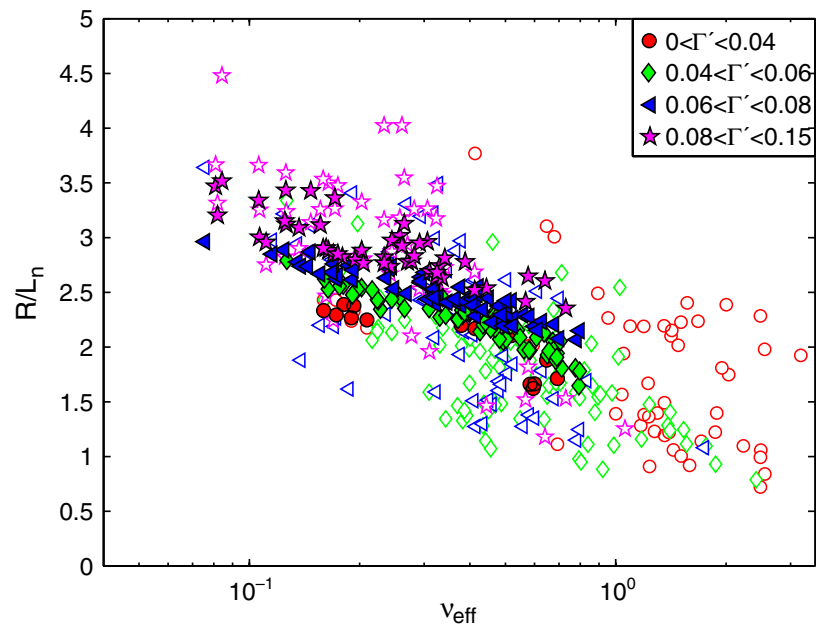

Figure 5. Comparison of simulation (un-filled symbols) with experimental results (filled symbols). Different symbols correspond to different $\Gamma^{\prime}$.

In figure $4(b)$ we show the comparison of $R / L_{\mathrm{n}}$ obtained from expression (5) with the values used as input for GS2. The RMS deviation between real and approximated $R / L_{\mathrm{n}}$ values is $\sim 0.066$ and the maximum deviation found is 0.23 (at $R / L_{\mathrm{n}} \sim 1.0$ ).

Note that $\Gamma_{\mathrm{GS} 2}$ and $\Gamma^{\prime}$ have the same definition, so we can substitute $\Gamma^{\prime}$ values into (5) to make a direct comparison with the experiment.

In figure 5 the normalized density gradient values obtained from expression (5) using experimental $\Gamma^{\prime}, v_{\text {eff }}$ and $T_{\mathrm{i}} / T_{\mathrm{e}}$ parameters are plotted in colour on top of the real measured $R / L_{\mathrm{n}}$ (grey points). There is good quantitative agreement between experimental and predicted values and a similar scaling with effective collisionality is apparent. The coloured points in figure 5 represent different ranges of the $\Gamma^{\prime}$ values, which can be seen to also have an effect, albeit a smaller one. 
To compare the GS2 simulation results with the linear empirical scaling expressions, a few transformations are required. The empirical scalings obtained in this work contain either $\Gamma^{\prime}$ or $T_{\mathrm{i}} / T_{\mathrm{e}}$, but not both of them, due to their high correlation in the experiments considered here. So we must remove $\Gamma^{\prime}$ or $T_{\mathrm{i}} / T_{\mathrm{e}}$ from (5) by using the relations (3), which describe their relationship:

$$
\begin{aligned}
R / L_{\mathrm{n}} & \sim\left(\frac{0.119+0.0656 T_{\mathrm{i}} / T_{\mathrm{e}}-0.145 \sqrt{\nu_{\mathrm{eff}}}}{0.0305-0.0081 \sqrt{\nu_{\mathrm{eff}}}}\right)^{2 / 3} \\
& \sim\left(\frac{0.1282+0.6028 \Gamma^{\prime}-0.1157 \sqrt{\nu_{\mathrm{eff}}}}{0.0305-0.0081 \sqrt{\nu_{\mathrm{eff}}}}\right)^{2 / 3} .
\end{aligned}
$$

To obtain a linear form, we use the following approximations: $0.0305-0.0081 \sqrt{v_{\mathrm{eff}}} \approx 0.0263$,

$$
R / L_{\mathrm{n}}^{3 / 2} \approx 2.106 R / L_{\mathrm{n}}-1.286
$$

for $v_{\text {eff }}=0.1-0.5$ and $R / L_{\mathrm{n}}=1.0-3.0$. Expression (6) transforms into

$$
R / L_{\mathrm{n}}=2.76-2.62 v_{\mathrm{eff}}^{1 / 2}+1.18 T_{\mathrm{i}} / T_{\mathrm{e}}
$$

and

$$
R / L_{\mathrm{n}}=2.92-2.09 v_{\mathrm{eff}}^{1 / 2}+10.83 \Gamma^{\prime}
$$

Comparing (7) with the empirical scaling (2) one can see that the GS2 simulations show a parametric dependence very similar to the experimental one.

An important observation from GS2 simulations is the opposite effect of the parameters $\Gamma^{\prime}$ and $T_{\mathrm{i}} / T_{\mathrm{e}}$ on the density profile. Each of those parameters has a strong impact on the density gradient, but due to their cross-correlation in our experimental conditions, described by expression (3), the empirically derived dependences are smaller and may be misleading. The coefficient for the $T_{\mathrm{i}} / T_{\mathrm{e}}$ parameter even changes the sign when we go from (5) to (6).

As a consequence, the empirical scaling $(2,3)$ should not be extrapolated to plasma devices where the $T_{\mathrm{i}} / T_{\mathrm{e}}$ parameter variation is large and/or does not correlate with core particle fuelling. That can explain a dramatic difference between the density peaking observed in JET and AUG H-mode plasmas and ECRH heated H-mode case on TCV [15] (with $T_{\mathrm{i}} / T_{\mathrm{e}} \ll 1$ and vanishing core particle source), where density peaking is considerably higher for the same collisionalities, compared with the scalings derived in [2-4].

\section{Simulation results for different $R / L_{T_{\mathrm{i}}}$ values}

In the above, we considered only the simulations with $R / L_{T_{\mathrm{e}}}=$ $R / L_{T_{\mathrm{i}}}=6.0$. In figure 6 we present the simulation results for $T_{\mathrm{i}} / T_{\mathrm{e}}=1, R / L_{\mathrm{n}}=1.8,2.3,2.8$ and $R / L_{T_{\mathrm{i}}}=6.0$, 7.0, 8.0, 9.0. The general tendency is that for higher $R / L_{T_{\mathrm{i}}}$ the dependence of $\Gamma^{\prime}$ on $v_{\text {eff }}$ weakens. The dependence of $\Gamma^{\prime}$ on $R / L_{T_{\mathrm{i}}}$ is somewhat complex. At low $\Gamma^{\prime}$ values it increases with $R / L_{T_{\mathrm{i}}}$ and at intermediate $\Gamma^{\prime}$ it changes sign. In other words, plasmas with high core particle source increase their density gradient with increasing $R / L_{T_{\mathrm{i}}}$, while plasmas with low or no core particle sources have the opposite behaviour. Interestingly, there is a turning point $\left(R / L_{\mathrm{n}}=2.3\right.$, $\Gamma^{\prime} \sim 0.05, v_{\text {eff }} \sim 0.25$ ) where $\Gamma^{\prime}$ and density gradient are independent of $R / L_{T_{\mathrm{i}}}$.

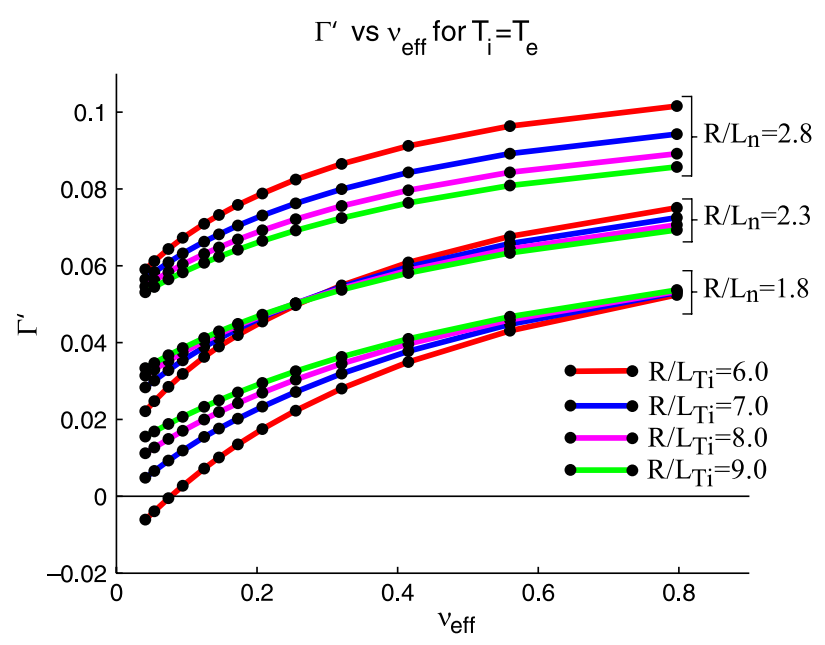

Figure 6. Simulation results for different $R / L_{T_{\mathrm{i}}}$ values.

To estimate the effect of different $R / L_{T_{\mathrm{i}}}$ values on the predicted density gradients we will construct the functions $R / L_{\mathrm{n}}=f\left(\Gamma^{\prime}, T_{\mathrm{i}} / T_{\mathrm{e}}, v_{\mathrm{eff}}\right)$ by analogy with (5) for other $R / L_{T_{\mathrm{i}}}$ values $(7.0,8.0,9.0)$ and compare their output for JET experimental $\Gamma^{\prime}, T_{\mathrm{i}} / T_{\mathrm{e}}$ and $\nu_{\text {eff }}$ parameters.

$R / L_{T_{\mathrm{i}}}=7.0$,

$$
\begin{gathered}
R / L_{\mathrm{n}}=\left(\frac{0.12+\Gamma^{\prime}-0.0511 T_{\mathrm{i}} / T_{\mathrm{e}}-0.082 \sqrt{\nu_{\mathrm{eff}}}}{0.0247-0.0057 \sqrt{\nu_{\mathrm{eff}}}}\right)^{2 / 3}, \\
\mathrm{RMS}=0.046, \mathrm{MAX}=0.18
\end{gathered}
$$

$R / L_{T_{\mathrm{i}}}=8.0$,

$$
\begin{gathered}
R / L_{\mathrm{n}}=\left(\frac{0.101+\Gamma^{\prime}-0.049 T_{\mathrm{i}} / T_{\mathrm{e}}-0.0702 \sqrt{\nu_{\mathrm{eff}}}}{0.0208-0.0042 \sqrt{\nu_{\mathrm{eff}}}}\right)^{2 / 3}, \\
\mathrm{RMS}=0.045, \mathrm{MAX}=0.21,
\end{gathered}
$$

$R / L_{T_{\mathrm{i}}}=9.0$

$$
\begin{gathered}
R / L_{\mathrm{n}}=\left(\frac{0.087+\Gamma^{\prime}-0.047 T_{\mathrm{i}} / T_{\mathrm{e}}-0.062 \sqrt{\nu_{\mathrm{eff}}}}{0.018-0.0032 \sqrt{\nu_{\mathrm{eff}}}}\right)^{2 / 3}, \\
\mathrm{RMS}=0.05, \mathrm{MAX}=0.23
\end{gathered}
$$

In figure 7 we plot the expected $R / L_{\mathrm{n}}$ values for various ion temperature gradients obtained with expressions (8)-(10) as a function of the values expected for $R / L_{T_{\mathrm{i}}}=6$, our base case assumption.

The difference is noticeable at higher density gradients where $R / L_{\mathrm{n}}$ increases with $R / L_{T_{\mathrm{i}}}$. At intermediate gradient values more typical for JET, there is no difference between various $R / L_{T_{\mathrm{i}}}$ cases and at the lowest $R / L_{\mathrm{n}}$ a weak inverse tendency is observed. The cross-correlation coefficient between $R / L_{\mathrm{n}}$ values calculated by formulas (5), (8)-(10) with JET experimental parameters and corresponding $R / L_{T_{\mathrm{i}}}$ values 6.0-9.0 gives a coefficient of 0.18 for the cases $v_{\text {eff }}<0.5$ which were used in deriving the empirical dependences. So we conclude that from the GS2 simulations we do not expect any noticeable $R / L_{T_{\mathrm{i}}}$ dependence for the considered JET plasma conditions. This is consistent with our observations, for which we find a coefficient equal to 0.08 (table 1). 


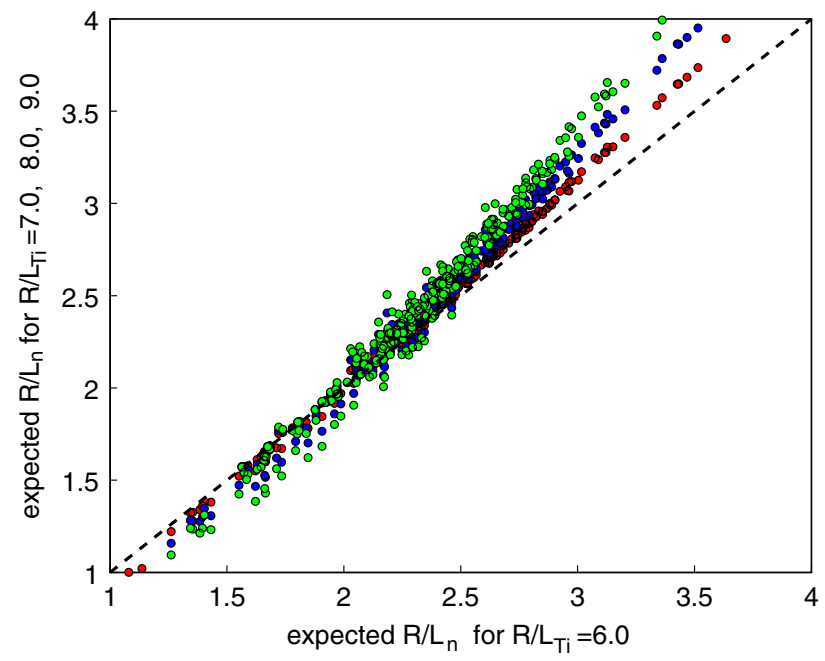

Figure 7. Expected $R / L_{\mathrm{n}}$ values in JET database for $R / L_{T_{\mathrm{i}}}=7.0$, $8.0,9.0$ with respect to initial $R / L_{T_{\mathrm{i}}}=6.0$ case.

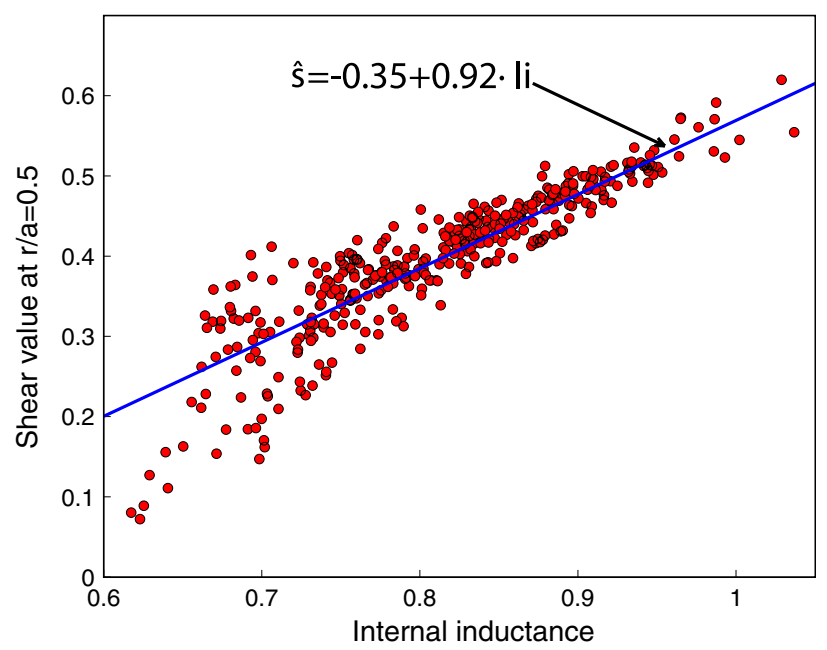

Figure 8. $\hat{s}$ at $r / a=0.5$ versus $l_{\mathrm{i}}$ values in JET database.

\section{Shear dependence in simulations and experiment}

In the empirical scaling $(1,2)$ we used the internal inductance as a characteristic of safety factor profile, since this value is the easiest to obtain. In the simulations only local values of magnetic shear $\hat{s}$ can be used, so we need to establish a relation between $l_{\mathrm{i}}$ and $\hat{s}=\mathrm{d} q / \mathrm{d} r \cdot r / q$ to compare the GS2 results with the experiment. In our database the $q$ profile was taken from the equilibrium code EFIT. This is a very basic estimation, but only this one is available on a routine basis.

In figure 8 we show that this relationship in the database can be fitted as

$$
\hat{s}(0.5) \approx-0.35+0.92 l_{\mathrm{i}}
$$

To estimate the effect of $\hat{s}$ in the simulation results, a separate smaller set of GS2 runs was produced. Three parameters were varied: $R / L_{\mathrm{n}}(1.8,2.3,2.8,3.2), v_{\mathrm{eff}}$ (nine values between 0.03 and 0.8$)$ and $\hat{s}(0.0,0.3,0.6,0.9)$. An example of the simulation results is shown in figure 9. Higher shear always leads to lower particle fluxes needed to maintain the given density gradient,

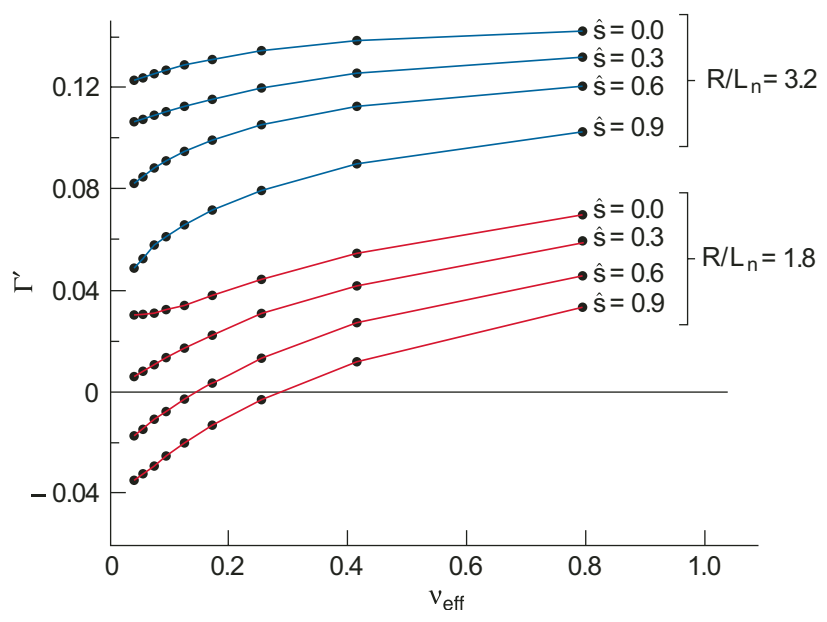

Figure 9. $\Gamma^{\prime}$ from GS2 simulations with different $R / L_{\mathrm{n}}$ and $\hat{s}$ inputs. $T_{\mathrm{i}} / T_{\mathrm{e}}=1, R / L_{T_{\mathrm{i}}}=R / L_{T_{\mathrm{e}}}=6.0$.

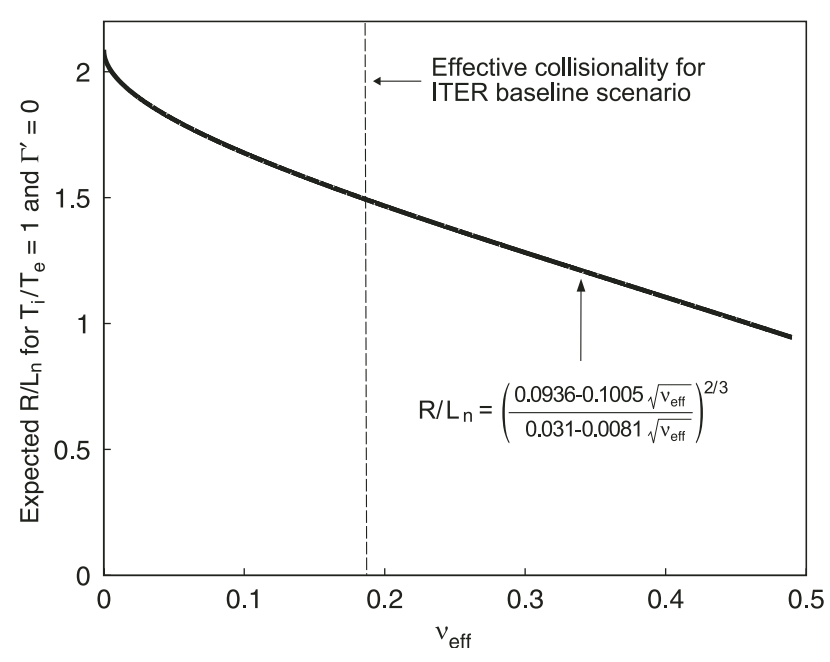

Figure 10. Expected $R / L_{\mathrm{n}}$ for $\Gamma^{\prime}=0$ and $T_{\mathrm{i}}=T_{\mathrm{e}}$.

so higher mid-radius shear and as a consequence higher $l_{i}$, should lead to stronger peaking.

To evaluate the effect of different $\hat{s}$ values on the density gradient expected from simulations, we performed a linear regression fit of $R / L_{\mathrm{n}}$ in the input data of GS2 runs versus collisionality, $\hat{s}$ and output $\Gamma^{\prime}$ values, with the result

$$
\begin{aligned}
& R / L_{\mathrm{n}} \approx 1.66 \pm 0.07-(1.1 \pm 0.1) v_{\mathrm{eff}}^{0.5}+(15.2 \pm 0.6) \Gamma^{\prime} \\
& \quad+(0.99 \pm 0.07) \hat{s} .
\end{aligned}
$$

That is a rough estimate compared with fits of the form (5) and gives an RMS of the deviation equal to 0.096 and maximum deviation 0.33 between regressed and the input $R / L_{\mathrm{n}}$ values. But it gives us an estimate of the predicted importance of shear on the density gradient:

$$
\frac{\partial\left(R / L_{\mathrm{n}}\right)}{\partial(\hat{s})} \sim 1.0
$$

Using the experimental relation (11) between $l_{\mathrm{i}}$ and $\hat{s}$ we transform it into

$$
\frac{\partial\left(R / L_{\mathrm{n}}\right)}{\partial\left(l_{\mathrm{i}}\right)} \sim 0.90 .
$$


AUG Pulse No: $13154, T_{e}=T_{i}, R / L_{n}=2$ with 64 toroidal modes $k_{\theta} \rho_{s}=(0.04252 .6783)$
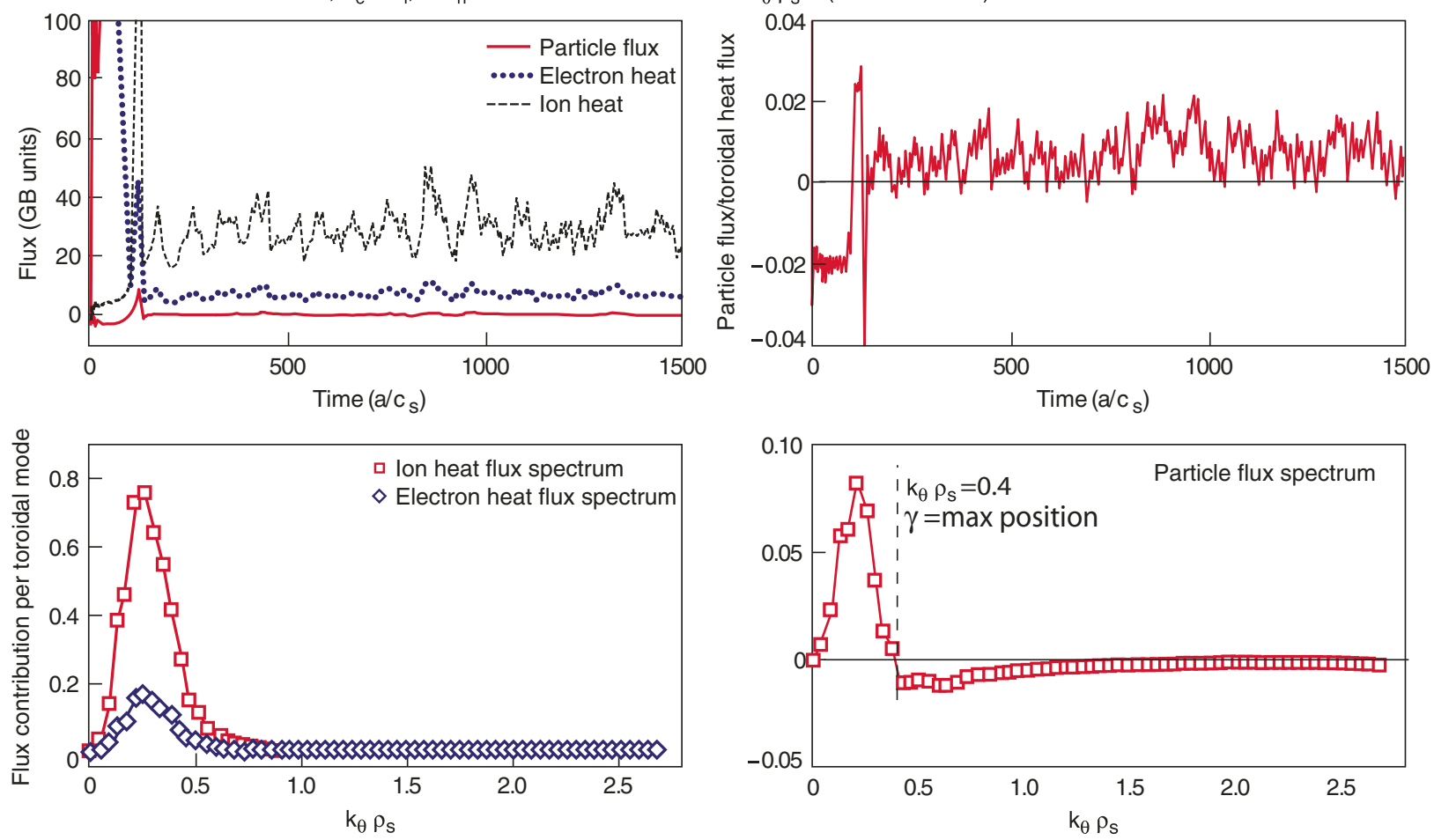

Figure 11. Non-linear GYRO simulation results top-left: heat and particle fluxes versus time; top-right: particle over heat flux versus time; bottom-left: $k_{y}$ spectrum for the heat flux and bottom-right: $k_{y}$ spectrum for the particle flux.

In the empirical database (2) these values are equal to $0.99 \pm 0.49$ for the $\left\{\left(v_{\text {eff }}\right)^{0.5} ; \Gamma^{\prime} ; l_{\mathrm{i}}\right\}$ fit and $1.28 \pm 0.51$ for the $\left\{v_{\text {eff }}^{0.5} ; T_{\mathrm{i}} / T_{\mathrm{e}} ; l_{\mathrm{i}}\right\}$ fit. We conclude that the GS2 linear simulations and JET observations agree within the error bars on the shear dependence too.

\section{Extrapolation of the simulations results to $\Gamma^{\prime}=0$, $T_{\mathrm{i}} / T_{\mathrm{e}}=1$ plasmas}

For ITER we may assume that $T_{\mathrm{i}} / T_{\mathrm{e}}=1$ and $\Gamma^{\prime}=0$. Fixing those values in expression (5) we can calculate the expected density gradient as a function of collisionality (see figure 10). For the collisionality value $v_{\text {eff }} \sim 0.19$, expected for ITER baseline $\mathrm{H}$-mode scenario, the expected value of the density gradient is $R / L_{\mathrm{n}}(\mathrm{ITER}) \sim 1.5$.

That is slightly lower than evaluated from empirical scaling (2), which predicts the gradient to be in the range 2.0-3.0, depending on which scaling expression is used.

We should however keep in mind that according to GS2, both $\Gamma^{\prime}$ and $T_{\mathrm{i}} / T_{\mathrm{e}}$ play a role. An experimental extrapolation based on a database, where as in the present one, these two quantities are strongly correlated, is therefore likely to suffer from uncertainties.

\section{Comparison with previous simulation results and non-linear GYRO calculations}

A study of the GS2 simulations of particle transport in tokamaks was already done in [16]. According to these results, the particle flux produced by ITG turbulence is directed outwards for all realistic collisionality values, which was in contradiction with the experimental observations. The main difference between the two studies (apart from the simulation plasma parameters) is the part of $k_{y}$ spectrum which was considered. In the previous work the focus was on linear modes with maximum $\gamma /\left\langle k_{\perp}^{2}\right\rangle$, which corresponds to $k_{y} \sim 0.1-0.2$. This quasi-linear method was motivated by nonlinear simulations of TEM turbulence in the collisionless limit [17], which identified the peak of heat and particle transport to match the maximum of $\gamma /\left\langle k_{\perp}^{2}\right\rangle$.

The simulations with the non-linear GYRO $[18,19]$ code have shown that in a collisional case, the actual non-linear spectrum is more complex and the modes with $\max \left(\gamma /\left\langle k_{\perp}^{2}\right\rangle\right)$ are not representative for the overall particle flux. In figure 11 we show the result of an electrostatic GYRO run with 2 kinetic species (electrons and deuterium) in a local Miller equilibrium, using a box size $L_{x}=82 \rho^{*}, L_{y}=148 \rho^{*}$ with resolution $\Delta x=0.58 \rho^{*}, 64$ toroidal modes between $k_{\theta} \rho_{s}(\mathrm{~min})=0.043$ and $k_{\theta} \rho_{s}(\max )=2.68$. The physical input parameters were $R / L_{T_{\mathrm{i}}}=R / L_{T_{\mathrm{e}}}=6.12, T_{\mathrm{i}} / T_{\mathrm{e}}=1, \hat{s}=1.12, q=1.33$, $\kappa=1.38, \delta=0.03, Z_{\text {eff }}=2$ (used for collision frequency calculations only), $v_{\mathrm{ei}}(\mathrm{GYRO})=v_{\mathrm{ei}}\left(a / c_{\mathrm{s}}\right)=0.018$, which correspond to $v_{\text {eff }} \sim 0.2$. For these plasma parameters the total particle flux calculated by GYRO is about zero, which means the input density gradient, $R / L_{\mathrm{n}}=2.0$, is stationary. This is in good agreement with the linear GS2 modelling based on a single wave number at the location of the fastest growing mode, which, for the input parameters used in the GYRO simulation delivers $R / L_{\mathrm{n}} \sim 2.17$ (if the difference in the local shear input parameter is taken into account using expression (13)). We observe that small wave numbers provide a contribution to the total particle flux which is directed strongly outwards, while large wave numbers provide an opposite contribution, directed 
inwards. A linear model based exclusively on the behaviour at small wave numbers, in the range $k_{\theta} \rho_{s}<0.25$, where $\gamma /\left\langle k_{\perp}^{2}\right\rangle$ is usually maximum, neglects the inward contribution at smaller scales, and leads to overestimate the total particle flux in the outward direction already at very small values of collisionality. Within a quasi-linear model based on a single wave number, which is practical for the application over extended datasets like in the present work, the location of the maximum growth rate is better suited to provide a description which is closer to the total particle flux obtained in the nonlinear saturated state. A deeper investigation of the non-linear behaviour over a collisionality range goes beyond the purpose of this work and will be presented in a separate publication [20].

\section{Conclusions}

On the basis of JET H-mode experiments we were able to find empirical relations which describe density peaking as a function of $\nu_{\mathrm{eff}}, T_{\mathrm{i}} / T_{\mathrm{e}}$, particle sources $\left(\Gamma^{\prime}\right)$ and $l_{\mathrm{i}}$. The general conclusions remain the same as in previous publications based on earlier experiments [1-5]. The effective collisionality plays the most important role in the density gradient formation. Extrapolation of the current experimental observations in JET to ITER parameters, predicts moderately peaked density profile for the baseline $\mathrm{H}$-mode scenario, which may be expected to improve fusion performance, as reported in [14].
We performed numerous simulations with the GS2 code, using the mode with maximum growth rate and varying the parameters involved in empirical scaling relations. The simulation results were abstracted in the form of simple expressions (5), (8)-(10). By equating $\Gamma_{\mathrm{GS} 2}$ to $\Gamma^{\prime}$, we made the assumption that all particle and ion heat fluxes are driven only by turbulent drift modes. The stationary density gradients predicted by the linear GS2 simulations are in good agreement with the experimental data over the wide range of parameters, including the same dependences on $v_{\mathrm{eff}}, T_{\mathrm{i}} / T_{\mathrm{e}}, \Gamma^{\prime}$ and $l_{\mathrm{i}}$ (or the local shear) and a negligible effect of $R / L_{T_{\mathrm{i}}}$.

As far as the dependence on the collision frequency is concerned, non-linear collisional GYRO simulations show that the quasi-linear analysis based on the mode with maximum growth rate, as used here, is better suited for particle transport than that based on the mode where $\max \left(\gamma /\left\langle k_{\perp}^{2}\right\rangle\right)$ occurs, as used in [16]. The results of the comparisons presented in this paper are supportive of drift wave theory as a leading candidate for explaining particle transport in tokamaks.

\section{Acknowledgments}

The authors thank W. Dorland and M. Kotschenreuther for providing the gyrokinetic code GS2, as well as J. Candy and R.E. Waltz for providing the gyrokinetic code GYRO.This work was supported in part by the Swiss National Science Foundation.

\section{Appendix}

\begin{tabular}{|c|c|c|c|c|c|c|}
\hline Const & $\left(v_{\text {eff }}\right)^{0.5}$ & $\Gamma^{\prime}$ & $T_{\mathrm{i}} / T_{\mathrm{e}}$ & $\delta$ & $l_{\mathrm{i}}$ & RMS \\
\hline \multicolumn{7}{|c|}{ One-parameter fit for density peaking } \\
\hline $1.96 \pm 0.04$ & $-0.88 \pm 0.07$ & & & & & 0.100 \\
\hline $1.29 \pm 0.02$ & & $2.93 \pm 0.38$ & & & & 0.121 \\
\hline $1.20 \pm 0.06$ & & & $0.30 \pm 0.05$ & & & 0.131 \\
\hline $1.74 \pm 0.03$ & & & & $-0.78 \pm 0.1$ & & 0.122 \\
\hline $1.11 \pm 0.10$ & & & & & $0.50 \pm 0.12$ & 0.136 \\
\hline \multicolumn{7}{|c|}{ Two-parameter fit for density peaking } \\
\hline $1.78 \pm 0.05$ & $-0.74 \pm 0.07$ & $1.58 \pm 0.32$ & & & & 0.093 \\
\hline $1.74 \pm 0.05$ & $-0.81 \pm 0.07$ & & $0.18 \pm 0.04$ & & & 0.094 \\
\hline $1.96 \pm 0.04$ & $-0.85 \pm 0.10$ & & & $-0.04 \pm 0.12$ & & 0.100 \\
\hline $1.79 \pm 0.09$ & $-0.84 \pm 0.07$ & & & & $0.19 \pm 0.09$ & 0.099 \\
\hline $1.58 \pm 0.08$ & & $6.67 \pm 1.01$ & $-0.53 \pm 0.13$ & & & 0.115 \\
\hline $1.53 \pm 0.04$ & & $2.54 \pm 0.33$ & & $-0.67 \pm 0.09$ & & 0.103 \\
\hline $0.79 \pm 0.09$ & & $3.2 \pm 0.34$ & & & $0.62 \pm 0.1$ & 0.108 \\
\hline $1.44 \pm 0.06$ & & & $0.28 \pm 0.05$ & $-0.76 \pm 0.09$ & & 0.109 \\
\hline $0.53 \pm 0.11$ & & & $0.39 \pm 0.05$ & & $0.73 \pm 0.11$ & 0.115 \\
\hline $1.61 \pm 0.12$ & & & & $-0.72 \pm 0.12$ & $0.15 \pm 0.12$ & 0.121 \\
\hline \multicolumn{7}{|c|}{ Three-parameter fit for density peaking } \\
\hline $1.78 \pm 0.07$ & $-0.73 \pm 0.08$ & $1.78 \pm 0.98$ & $-0.03 \pm 0.12$ & & & 0.093 \\
\hline $1.77 \pm 0.05$ & $-0.63 \pm 0.10$ & $1.68 \pm 0.32$ & & $-0.16 \pm 0.11$ & & 0.092 \\
\hline $1.43 \pm 0.10$ & $-0.63 \pm 0.07$ & $1.92 \pm 0.32$ & & & $0.34 \pm 0.09$ & 0.089 \\
\hline $1.64 \pm 0.07$ & & $4.43 \pm 0.96$ & $-0.26 \pm 0.13$ & $-0.61 \pm 0.09$ & & 0.102 \\
\hline $0.98 \pm 0.14$ & & $4.84 \pm 1.02$ & $-0.24 \pm 0.14$ & & $0.53 \pm 0.11$ & 0.107 \\
\hline $1.73 \pm 0.06$ & $-0.72 \pm 0.10$ & & $0.19 \pm 0.04$ & $-0.15 \pm 0.11$ & & 0.093 \\
\hline $1.00 \pm 0.13$ & & & $0.34 \pm 0.05$ & $-0.58 \pm 0.10$ & $0.42 \pm 0.11$ & 0.104 \\
\hline $1.78 \pm 0.10$ & $-0.87 \pm 0.10$ & & & $0.05 \pm 0.13$ & $0.21 \pm 0.1$ & 0.099 \\
\hline $1.31 \pm 0.12$ & $-0.70 \pm 0.07$ & & $0.25 \pm 0.04$ & & $0.39 \pm 0.09$ & 0.089 \\
\hline $1.00 \pm 0.13$ & & & $0.34 \pm 0.05$ & $-0.58 \pm 0.10$ & $0.42 \pm 0.11$ & 0.104 \\
\hline \multicolumn{7}{|c|}{ Four-parameter fit for density peaking } \\
\hline $1.29 \pm 0.15$ & & $3.78 \pm 0.97$ & $-0.14 \pm 0.13$ & $-0.50 \pm 0.10$ & $0.31 \pm 0.11$ & 0.100 \\
\hline $1.30 \pm 0.12$ & $-0.71 \pm 0.09$ & & $0.25 \pm 0.04$ & $0.01 \pm 0.12$ & $0.40 \pm 0.10$ & 0.089 \\
\hline $1.44 \pm 0.11$ & $-0.62 \pm 0.10$ & $1.91 \pm 0.32$ & & $-0.02 \pm 0.12$ & $0.33 \pm 0.09$ & 0.089 \\
\hline $1.35 \pm 0.13$ & $-0.66 \pm 0.08$ & $0.93 \pm 0.96$ & $0.13 \pm 0.12$ & & $0.37 \pm 0.09$ & 0.089 \\
\hline $1.77 \pm 0.07$ & $-0.63 \pm 0.11$ & $1.87 \pm 0.98$ & $-0.03 \pm 0.12$ & $-0.16 \pm 0.11$ & & 0.092 \\
\hline
\end{tabular}




\section{References}

[1] Angioni C. et al 2003 Phys. Rev. Lett. 90205003

[2] Weisen H. et al 2005 Nucl. Fusion 45 L1-L4

[3] Weisen H. et al 2006 Plasma Phys. Control. Fusion 48 A457-66

[4] Angioni C. et al 2007 Nucl. Fusion 47 1326-35

[5] Greenwald M. et al 2007 Nucl. Fusion 47 L26-9

[6] Takenaga H. et al 2008 Nucl. Fusion 48075004

[7] Challis CD et al 1989 Nucl. Fusion 29563

[8] Goldston R.J. et al 1981 J. Comput. Phys. 4361

[9] Weisen H. et al 2004 Plasma Phys. Control. Fusion 46 751-65

[10] Stix T.H. 1972 Plasma Phys. 14 367-84

[11] Mukhovatov V. et al 2003 Nucl. Fusion 43 942-8

[12] Kotschenreuther M., Rewoldt G. and Tang W.M. 1995 Comput. Phys. Commun. $\mathbf{8 8} 128$
[13] Dorland W. et al 2000 Phys. Rev. Lett. 8526

[14] Weisen H. et al 2006 21st IAEA Fusion Energy Conf. (Chengdu, China) EX8-4 http://www-pub.iaea.org/MTCD/ Meetings/FEC2006/ex_8-4.pdf, http://www-pub.iaea.org/ MTCD/Meetings/FEC2006Presentations/Presentations/ 20-oct-06/ talk_EX8-4.ppt

[15] Porte L. et al 2007 Nucl. Fusion 47 952-60

[16] Angioni C. et al 2005 Phys. Plasmas 12112310

[17] Jenko F., Dannert T. and Angioni C. 2005 Plasma Phys. Control. Fusion 47 B195-206

[18] Candy J. and Waltz R.E. 2003 J. Comput. Phys. 186545

[19] Candy J. and Waltz R.E. 2003 Phys. Rev. Lett. 91045001

[20] Angioni C. et al 2009 Particle pinch and collisionality in gyrokinetic simulations of tokamak plasma turbulence Phys. Plasmas submitted 